\title{
Metastasis to breast from ovarian cancer and primary ovarian cancer concurrently diagnosis
}

\author{
Moon Il Lee ${ }^{1}$, Youn Joo Jung ${ }^{2} \wedge$, Dong Il Kim²^, Hyun-June Paik ${ }^{2}$, Seungju Lee ${ }^{2}$, Chang Shin Jung ${ }^{2}$, \\ Jee Yeon Kim ${ }^{3}$, Hyun Yul Kim ${ }^{2} \wedge$ \\ ${ }^{1}$ Breast Surgery, Hwamyoung Ilsin Christian Hospital, Pusan, Korea; ${ }^{2}$ Department of Surgery, Pusan National University Yangsan Hospital, Yangsan, \\ Korea; ${ }^{3}$ Department of Pathology, Pusan National University Yangsan Hospital, Yangsan, Korea \\ Correspondence to: Hyun Yul Kim. Department of Surgery, Pusan National University Yangsan Hospital, 20, Geumo-ro, Mulgeum-eup, Yangsan-si, \\ Gyeongsangnam-do, Korea. Email: isepa102@naver.com.
}

\begin{abstract}
Metastasis to breast from ovarian cancer is very rare, and it is very difficult to distinguish the primary breast cancer from the secondary one. In our case, a 61-year-old woman presented to the hospital with complaints of lower abdominal pain and distention. Abdominal-pelvic computed tomography (CT) imaging revealed a solid ovarian cystic tumor with numerable ascites in the abdominal cavity and disseminated peritoneal carcinomatosis. To confirm the presence of metastasis in other organs, a preoperative imaging studies including chest CT was performed. Chest CT imaging revealed an enlargement of both the axillary lymph nodes (r/o metastatic lymphadenopathy) and breast imaging studies (breast sonography and mammography) revealed masses penetrated the pectoralis major muscle in the left breast and metastatic lymphadenopathy in Lt axilla. The core needle biopsy result of breast lesion showed unknown origin carcinoma with micropapillary features and no psammoma bodies. So, we could not distinguish primary breast cancer and breast metastasis from ovarian cancer because breast metastasis from ovary could be contained psammoma bodies, generally. Although the possibility of primary breast cancer could not be ruled out absolutely, the neoadjuvant chemotherapy included Paclitaxel and Carboplatin is known to be effective for both breast and ovarian cancer. So after neoadjuvant chemotherapy, mastectomy concurrently with ovarian cancer surgery was performed. The histopathologic features of the ovaries and breast were similar and psammoma bodies also could be observed, which were not detected in previous core needle biopsied specimen. And lymph nodes from the breast and the ovaries also showed consistent findings. Combined with histopathologic report and medical records, the final diagnosis confirmed as a metastasis of ovarian cancer to breast. After surgery, the patient has continued with metastatic ovarian cancer specific treatment. Breast metastatic tumors must be distinguished from primary breast tumors to avoid any unnecessary surgery. It might be difficult to diagnosis precisely by using only core needle biopsy because of inadequate specimen volume. Although surgical resection may be effective for both diagnosis and palliative treatment, further studies focused on less invasive biopsy are needed for exact histopathological diagnosis.
\end{abstract}

Keywords: Ovarian cancer; breast metastasis; case report

Submitted Aug 03, 2020. Accepted for publication Jan 15, 2021.

doi: $10.21037 / g s-20-640$

View this article at: http://dx.doi.org/10.21037/gs-20-640

^ ORCID: Youn Joo Jung 0000-0002-9647-8556; Dong Il Kim 0000-0001-9874-1322; Hyun-June Paik 0000-0002-2749-4165; Seungju Lee 0000-0001-5100-0017; Chang Shin Jung 0000-0002-2945-4191; Hyun Yul Kim 0000-0001-7717-7734. 


\section{Introduction}

Breast metastasis form other solid organs (ovary, cervix, and other pelvic cavity organs) are very rare. Metastatic breast cancer accounts for only approximately $0.33-$ $6.3 \%$ of all breast cancers, and then almost cases also are from melanomas, sarcomas, lung cancer, and renal carcinomas $(1,2)$. Because imaging studies (mammography, ultrasonographic images) have a low specificity, it is hard to distinguish the primary breast cancer from secondary breast cancer using by imaging studies alone. In comparison with primary breast cancer, metastatic or secondary breast cancer has a relatively poorer prognosis (3). Making an adequate therapeutic plan is also very difficult. So that treatment plan of breast metastasis focuses on tumor management by using systemic chemotherapy to avoid unnecessary surgery.

Especially, metastases to the breast from ovarian cancer are rare, and only a few cases have been reported globally since 1907 , when the first case was reported $(4,5)$. This report describes our experience with a patient who presented with metastasis to the breast from ovarian cancer. Usually, metastases to breast detected within 2 years from the initial diagnosis of primary ovarian cancer (6). But, our patient diagnosed with primary ovarian cancer and metastasis to the breast concurrently.

In addition, to the best of our knowledge, there are only a few reports for breast metastasis from ovary. But this case report that existing concurrently both breast metastasis from ovary and ovarian cancer when initial diagnosis might be the first. And it is strengths for our case report.

We present the following case in accordance with the CARE reporting checklist (available at http://dx.doi. org/10.21037/gs-20-640).

\section{Case presentation}

A 61-year-old woman presented to the hospital with complaints of lower abdominal pain and distention in august 2017. According to the Patient's family history, the older brother had gastric cancer. The patient had never been diagnosed with ovarian or breast cancer before.

Abdominal-pelvic computed tomography (CT) imaging revealed both a solid ovarian cystic tumor with numerable ascites in the abdominal cavity and disseminated peritoneal carcinomatosis (Figure 1). To confirm the presence of metastatic disease in other organs, a preoperative chest CT scanning was performed. This revealed an enlargement of both the axillary lymph nodes (r/o metastatic lymphadenopathy). The radiologist recommended further breast evaluation. Breast ultrasound and mammography revealed a mass near the retro mammary space in the left breast. This mass penetrated the pectoralis major muscle and metastatic lymphadenopathy was noted in both axillas (Figure 2A,B).

The core needle biopsy from breast lesion showed unknown originated carcinoma with micropapillary features with no psammoma body (Figure $3 A$ ). Immunohistochemical analysis of the tumor revealed the mass to be estrogen receptor (ER) negative, progesterone receptor $(\mathrm{PR})$ positive (Allred score 5), human epidermal growth factor 2 (HER2) negative (score 0 ), p5 3 protein positive, Wilms' tumor 1 (WT-1) positive, paired box gene 8 (PAX8) positive, GATA binding protein 3 (GATA3) negative, and with $\mathrm{Ki}-67$ index of $80 \%$.

Based on the histologic features of micropapillary formation with positive PR immunostaining, the possibility of primary breast cancer could not be ruled out absolutely. But, this might suggested the possibility of metastatic carcinoma from the ovary, rather than primary micropapillary carcinoma of the breast, considering ovarian cancer history.

The patient underwent neoadjuvant chemotherapy (Paclitaxel $175 \mathrm{mg} / \mathrm{BSA}$, Carboplatin 5 AUC, every three weeks for three cycles) prior to surgery. This chemotherapy is known to be effective for both breast and ovarian cancer. Under general anesthesia, total abdominal hysterectomy + bilateral salpingo-oophorectomy (TAH/BSO), low anterior resection (LAR) were performed in November 2017. And radical total mastectomy also performed for precise diagnosis and palliative purpose.

The histologic features of the right and left ovaries showed high grade serous carcinomas, measuring $8.8 \mathrm{~cm} \times$ $7.4 \mathrm{~cm}$ and $4.8 \mathrm{~cm} \times 3.7 \mathrm{~cm}$ in size, respectively (Figure $3 \mathrm{~B}$ ). Most of the ovary was replaced by a solid tumor mass with cystic area. The resected breast showed multiple tumor masses, ranging from 0.3 to $2.0 \mathrm{~cm}$ in size. The histologic features of the ovaries and right breast were similar to each other, with micropapillae and psammoma bodies, which were not detected in previous core needle biopsied specimen (Figure 3C). Immunostainings for PR, p53, WT1, and PAX8 were positive; however, ER and GATA3 were negative, and $\mathrm{Ki}-67$ index was $80 \%$. Lymph nodes from the breast and the ovaries, rectum, and omentum showed consistent findings. The final diagnosis was high grade serous carcinoma of the ovaries, and metastases to the left breast and rectum, including the regional lymph nodes. 
Adjuvant chemotherapy was administered (Paclitaxel $175 \mathrm{mg} / \mathrm{BSA}$, Carboplatin 5 AUC, every three weeks for six cycles) after surgery. We checked for side effects at each chemotherapy cycle. There were mild nausea, dyspnea and moderate tingling in both hand, foot. But the patient could tolerate it.

Positron emission tomography/computed tomography (PET/CT) was conducted In June 2018. This imaging showed residual tumor; hence, palliative chemotherapy (doxorubicin $40 \mathrm{mg} / \mathrm{BSA}$, bevacizumab $10 \mathrm{mg} / \mathrm{kg}$ ) was administered. After 4 cycles of palliative treatment, we discussed about prognosis with patient and she refused more treatment. Follow up has been lost since September 2018.

All procedures performed in studies involving human participants were in accordance with the ethical standards of

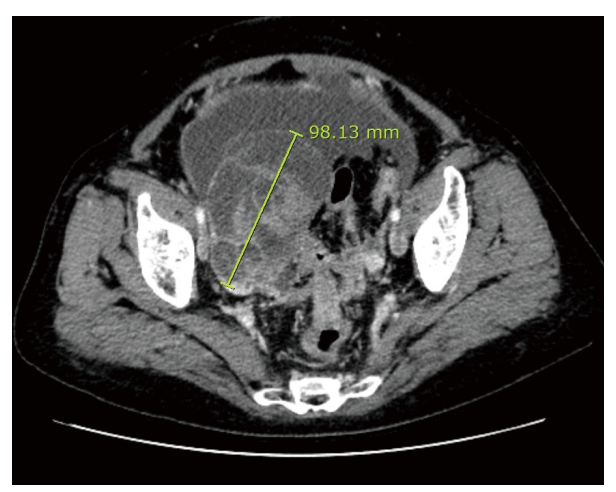

Figure 1 Abdominal-pelvic CT images of the ovarian cancer; note the larger, bilateral ovarian masses and ascites. the institutional and/or national research committee(s) and with the Helsinki Declaration (as revised in 2013). Written informed consent was obtained from the patient. This study was approved by the Institutional Review Board (IRB) of the Pusan National University Yangsan Hospital (IRB No. 05-2020-153).

\section{Discussion}

Breast cancer is the most frequently diagnosed primary cancer in women. Breast cancer almost always originates from the mammary glands, and most metastatic breast cancers originate from the contralateral breast (3). Advanced ovarian cancer typically demonstrates peritoneal dissemination or lymph node metastases in the abdominal cavity; distant metastases are rare. However, ovarian cancer occasionally metastasizes to the liver, pleura, or lung, and the prognosis of ovarian cancer patients with distant metastasis is poor (7).

Primary gynecological cancers that metastasize to the breast are rare. The overall incidence of primary gynecologic tumor metastases to the breast was $0.17 \%$, and among all the breast cancer cases, only $0.07 \%$ are mammary metastases from primary ovarian tumors (8).

Distinguishing primary breast cancer form metastatic breast cancer is very difficult because imaging findings are not specific and diagnostic (9). Generally, almost breast metastases are detected by mammography, and typically consist of a round and clear-margined mass. There are seldom unclear margins, and microlobulated
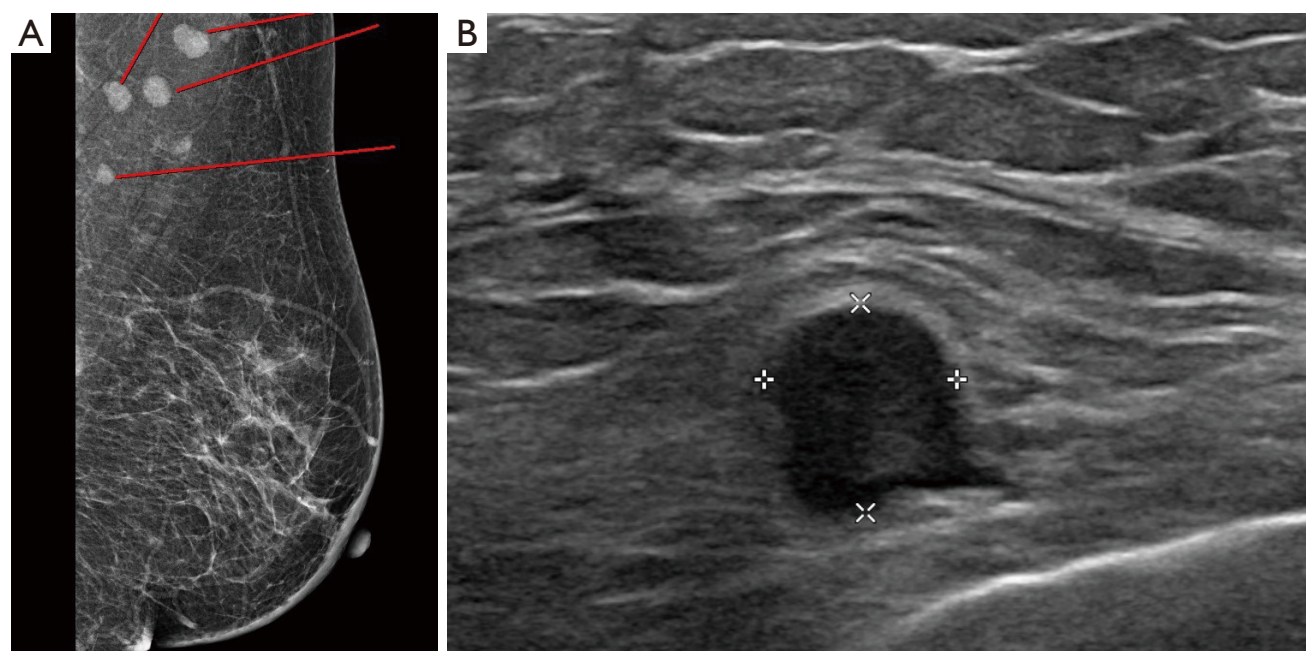

Figure 2 Radiologic findings. (A) Metastatic lymphadenopathy at mammography; Lt. axilla with multiple enlarged lymph nodes; (B) ultrasonography image revealing a regular-bordered round or oval shaped hypoechoic lesion. 

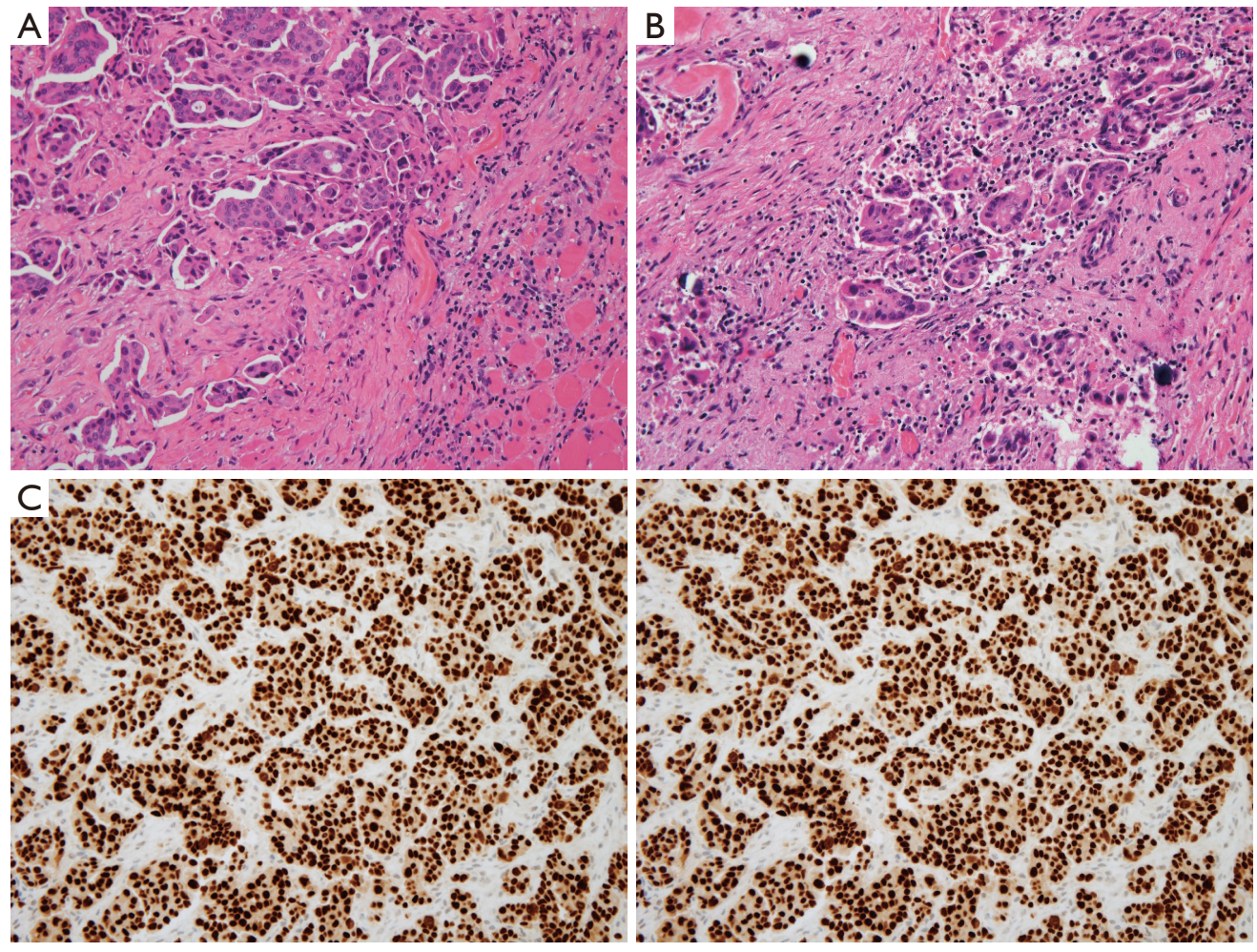

Figure 3 Radiologic findings. (A) Histologic features of the core needle biopsy of the breast tissue showing invasive carcinoma with micropapillary features without psammoma body (H\&E stain, $\times 100)$. (B) Microscopic features of the right ovary. High grade carcinoma with micropapillary features and tumor infiltrating lymphocytes (TIL) and psammoma bodies (H\&E stain, $\times 200)$; (C) (left) Immunohistochemical staining for PAX $-8(\times 100)$ and (right) W'T-1 $(\times 100)$ showed strong and diffuse positivity.

shaped masses are rare. Furthermore, there is generally no evidence of microcalcification or architectural distortion on mammography (2). Calcifications are not usually present in patients with metastases to breast from ovarian cancer because of the presence of psammomatous bodies (1013) and then most lesions of this type often founded in the subcutaneous layer of the breast mammary gland because of the rich blood supply to this region and axillary lymph node metastases are seldom revealed, compared with primary breast cancer (14).

The treatment and prognosis of primary breast cancer and metastasis to the breast from ovary are very different. Breast metastasis from ovarian cancer may disseminate widely and it is associated with a poor prognosis. The mean-survival time was previously reported to range from 13 days to 3.5 years (15-17). However, many patients have survived in less than one year after the diagnosis. Statistics on prognosis show that the 1-year survival rate is about $40 \%$ (12) the treatment of choice for metastasis from ovarian cancer is generalized systemic chemotherapy with appropriate chemotherapeutic agents (platinum based chemotherapy) and cytoreductive surgery to reduce tumor burden, whereas surgical management should be considered diagnostic and palliative (NCCN guidelines version 1.2020).

If the metastatic breast lesion is not larger or positioned in the deep tissue layers, and if there are no symptoms, palliative surgery also should not be considered. If only patients have a symptom, a mastectomy should be performed for only palliative purposes (18).

For these reasons, accurate and precise diagnosis is very necessary to make a treatment plan. The treatment plan should be determined considering combined data from an accurate medical history, physical examination, imaging studies analyses and definitive biopsy results. Therefore, surgeons could not perform unnecessary mastectomy.

When initial diagnosis, imaging studies revealed both ovary cancer and metastasis to breast concurrently in our case. There are other two possibilities which secondary ovarian cancer and primary breast cancer and double primary breast cancer and ovarian cancer as co-morbidities. 
Differentiating between primary and secondary ovarian cancer can be a difficult task. In hereditary conditions breast malignancies and primary ovarian cancer often coexist. BRCA1 and BRCA2 mutations are responsible for the concomitant occurrence of these two cancer types. Specifically, women with BRCA1 alterations have a high risk of developing breast (85\%) and ovarian (40-60\%) cancer, whilst women with BRCA2 mutations have a similar risk of breast cancer as with BRCA1 mutations and $15-30 \%$ probability of developing ovarian cancer $(19,20)$. The bare suggestion that only core needle biopsy results, it could not be possible to distinguish primary cancer from secondary cancer. Although the possibility of primary breast cancer could not be ruled out absolutely, we decided that the possibility of metastatic carcinoma from the ovary is higher than the one of primary breast cancer considering as a history of ovarian cancer. Metastatic breast cancer must be excluded by appropriate histopathological examination. Although the resection of the breast lesion does not positively affect the prognosis for metastasis to breast from ovary, it appears to be important to have an adequate specimen in order to establish the precise diagnosis. Follow above, the most common histological type of breast metastases from ovary would be serous papillary carcinoma contained psammoma bodies (8). But in our case, the core needle biopsy result of breast lesion revealed a micropapillary lesion with no psammoma bodies. It makes us unable to make an accurate diagnosis before surgery. So, radical mastectomy concurrently with ovarian cancer surgery was performed. This surgical intervention was very effective method for both treatment and diagnosis, because our patient have complained pain for breast lesion also and the specimen from mastectomy can present more precise and exact histopathological results for diagnosis.

In conclusion, this is the very rare case for breast metastasis from ovary and ovarian cancer concurrently when initial diagnosis. But if there are breast lesions concurrently with ovarian cancer, possibilities of metastasis to breast from ovary might be considered in patients with an ovarian cancer. It might be difficult to diagnosis precisely using only core needle biopsy because of inadequate specimen volume.

Further studies focused on less invasive biopsy for exact histopathological diagnosis are needed for accurate differentiation to avoid unnecessary surgery.

\section{Acknowledgments}

Funding: None.

\section{Footnote}

Reporting Checklist: The authors have completed the CARE reporting checklist. Available at http://dx.doi.org/10.21037/ gs-20-640

Conflicts of Interest: All authors have completed the ICMJE uniform disclosure form (available at http://dx.doi. org/10.21037/gs-20-640). The authors have no conflicts of interest to declare.

Ethical Statement: The authors are accountable for all aspects of the work in ensuring that questions related to the accuracy or integrity of any part of the work are appropriately investigated and resolved. All procedures performed in studies involving human participants were in accordance with the ethical standards of the institutional and/or national research committee(s) and with the Helsinki Declaration (as revised in 2013). Written informed consent was obtained from the patient. This study was approved by the Institutional Review Board (IRB) of the Pusan National University Yangsan Hospital (IRB No. 05-2020-153).

Open Access Statement: This is an Open Access article distributed in accordance with the Creative Commons Attribution-NonCommercial-NoDerivs 4.0 International License (CC BY-NC-ND 4.0), which permits the noncommercial replication and distribution of the article with the strict proviso that no changes or edits are made and the original work is properly cited (including links to both the formal publication through the relevant DOI and the license). See: https://creativecommons.org/licenses/by-nc-nd/4.0/.

\section{References}

1. Lee AH. The histological diagnosis of metastases to the breast from extramammary malignancies. J Clin Pathol 2007;60:1333-41.

2. Abbas J, Wienke A, Spielmann RP, et al. Intramammary metastases: comparison of mammographic and ultrasound features. Eur J Radiol 2013;82:1423-30.

3. Cormio G, Rossi C, Cazzolla A, et al. Distant metastases in ovarian carcinoma. Int J Gynecol Cancer 2003;13:125-9.

4. Sitzenfrey A. Mammakarzinom zwei jahre nach abdominaler radikaloperation wegen doppelseitigen carcinoma ovarii. Prag Med Wochenschr 1907;32:221-35.

5. Su F, Zeng Y, Jia W, et al. Breast metastasis of ovarian cancer: a case report. Chin J Oncol 2001;11. 
6. Klein RL, Brown AR, Gomez-Castro CM, et al. Ovarian cancer metastatic to the breast presenting as inflammatory breast cancer: a case report and literature review. J Cancer 2010;1:27-31.

7. Georgiannos SN, Chin J, Goode AW, et al. Secondary neoplasms of the breast: a survey of the 20th century. Cancer 2001;92:2259-66.

8. Moore DH, Wilson DK, Hurteau JA, et al. Gynecologic cancers metastatic to the breast. J Am Coll Surg 1998;187:178-81.

9. Bitencourt AGV, Gama RRM, Graziano L, et al. Breast metastases from extramammary malignancies: multimodality imaging aspects. Br J Radiol 2017;90:20170197.

10. Sippo DA, Kulkarni K, Carlo PD, et al. Metastatic disease to the breast from extramammary malignancies: a multimodality pictorial review. Curr Probl Diagn Radiol 2016;45:225-32.

11. Balaji R, Ramachandran K, Anila KR. Ovarian carcinoma metastasis to the breast and imaging features with histopathologic correlation: a case report and review of the literature. Clin Breast Cancer 2009;9:196-8.

12. Loredo DS, Powell JL, Reed WP, et al. Ovarian carcinoma metastatic to breast: a case report and review of the literature. Gynecol Oncol 1990;37:432-6.

Cite this article as: Lee MI, Jung YJ, Kim DI, Paik HJ, Lee S, Jung CS, Kim JY, Kim HY. Metastasis to breast from ovarian cancer and primary ovarian cancer concurrently diagnosis. Gland Surg 2021;10(5):1806-1811. doi: 10.21037/gs-20-640
13. Yamasaki H, Saw D, Zdanowitz J, et al. Ovarian carcinoma metastasis to the breast case report and review of the literature. Am J Surg Pathol 1993;17:193-7.

14. Mun SH, Ko EY, Han BK, et al. Breast metastases from extramammary malignancies: typical and atypical ultrasound features. Korean J Radiol 2014;15:20-8.

15. Ozgüroğlu M, Ersavaşti G, Ilvan S, et al. Bilateral inflammatory breast metastases of epithelial ovarian cancer. Am J Clin Oncol 1999;22:408-10.

16. Micha JP, Goldstein BH, Epstein HD, et al. Ovarian cancer metastatic to the breast. Gynecol Oncol 2006;102:386-90.

17. Maffini F, Bozzini A, Casadio C, Ovarian serous papillary carcinoma, metastatic to intramammary lymph-node mimic a primary breast carcinoma on RX mammography. Breast J 2012;18:484-5.

18. Hajdu SI, Urban JA. Cancers metastatic to the breast. Cancer 1972;29:1691-6.

19. Souza FF, Katkar A, den Abbeele AD, et al. Breast angiosarcoma metastatic to the ovary. Case Rep Med 2009;2009:381015.

20. Li W, Wang H, Wang J, et al. Ovarian metastases resection from extragenital primary sites: outcome and prognostic factor analysis of 147 patients. BMC Cancer 2012;12:278. 